\title{
Fluorescence induite par laser sur les tissus profonds du cerveau du rat : application à l'étude du cycle veille-sommeil
}

\author{
S.J. Mottin, P. Laporte, R. Cespuglio* et M. Jouvet* \\ Laboratoire de Traitement du Signal et Instrumentation, URA 842 du CNRS, Université Jean \\ Monnet, 42023 Saint-Etienne, France \\ * Département de Médecine Expérimentale, INSERM U 52, 69373 Lyon, France
}

\begin{abstract}
A nanosecond time resolved fluorescence, derived from the nucleus Raphe dorsalis (nRD) of the rat is reported. A single optical fiber is used for the transmission of the subnanosecond nitrogen laser pulse $(337 \mathrm{~nm})$ and the collection of the NADH brain fluorescence at $460 \mathrm{~nm}$. This method also combined with polygraphic recordings, is used in animals under long-term chronic conditions and unanesthetized. Thoughout the Sleep-Wake cycle and versus the waking state (W), the NADH peak measured increases during slow wave sleep (SWS) and paradoxical sleep (PS) or during PS only. The variations observed are dependent upon the optical fiber location within the $\mathrm{nRD}$. The existence of oxidation mechanisms for sleep triggering and maintenance are discussed.

Résumé: Un capteur chimique à fibre optique monofibre monobrin est utilisé pour transmettre le rayonnement d'excitation d'un laser azote et collecter la fluorescence liée au NADH cérébral. Cette méthode fluorimétrique couplée aux enregistrements polygraphiques est réalisée chez le rat en condition chronique long-terme. Au niveau du noyau du raphé dorsal (nRD) la fluorescence mesurée a $460 \mathrm{~nm}$ présente une faible augmentation pour le sommeil paradoxal (SP) ainsi que dans certaines sous-structures pour le sommeil a onde lente (SL) par rapport au niveau enregistré pendant l'éveil; cette variation physiologique pourrait refléter l'activité dendritique. Ces mesures suggèrent une variation du métabolisme pendant le SP. La nature des mécanismes neurochimiques qui soustendent le SP est discutée.
\end{abstract}

\section{INTRODUCTION}

Les capteurs chimiques à fibre optique (FOCS) ont commencé à apparaître à la fin des années soixante-dix sous la forme d'ajout de fibre optique à des appareils de mesure optique classique (fluorimetrie en cuvette) [1], [2]. A partir du milieu des années quatrevingt ils se sont diversifiés en plusieurs catégories: - les capteurs extrinsèques (la fibre optique restant passive); - semi-intrinsèques (la grandeur optique mesurée découle d'une réaction chimique localisée à la surface des fibres); - intrinsèques (les phénomènes de guide de lumiere interviennent directement dans la mesure chimique) [3].Les secteurs d'applications sont majoritairement le domaine de la chimie analytique, lié à l'environnement, et le domaine biomédical. 
Dans ce dernier, les technologies des capteurs chimiques utilisés in vivo sont restreintes aux capteurs électrochimiques (micro-électrodes a fibre de carbone), et aux capteurs à perméation (microdialyse))

Pour l'étude des mécanismes neurochimiques du cycle veille-sommeil, qui doit être nécessairement réalisée en conditions chronique long-terme, ces méthodes présentent des limites [4].

Il est vrai pour toute discipline scientifique que la progression du niveau des connaissances est étroitement liée aux performances des outils utilisés. L'évolution de la compréhension des mécanismes du cycle veille-sommeil, illustre bien cet aspect des choses. L'introduction de l'électroencéphalographie dans les années 30 a permis une quantification objective des états de vigilance. Par la suite, la réalisation de lésions, de stimulations au sein du cerveau ont permis la localisation des structures impliquées dans la genèse de l'éveil ou du sommeil. La mise au point des techniques d'histofluorescence et l'apport de la pharmacologie ont été aussi déterminants puisque parallèlement à l'identification de certaines substances biochimiques au sein du cerveau, il a été possible d'agir sur leur synthèse, leur dégradation, ou les récepteurs affectés dans la transmission d'un message biochimique.

Dans ce contexte et à travers l'usage de ces différents outils, la Sérotonine (5-HT), une amine identifiée dans les groupes cellulaires du noyau du raphé dorsal (nRD), a pu être suggérée comme le neurotransmetteur directement responsable du sommeil lent et du déclenchement du sommeil paradoxal. En effet, l'inhibition de sa synthèse par la p.chlorophénylalanine (PCPA) induit une insomnie totale qui est accompagnée par une diminution de son contenu endogene et de son immunofluorescence tissulaire évalués post-mortem. Ladministration secondaire de 5-HTP (5-Hydroxytryptophane), le précurseur immédiat de la 5-HT qui est rapidement transformé en Sérotonine, restaure selon une relation dose effet l'ensemble des paramètres évoqués ci-dessus [5]. Ces faits expérimentaux, solidement établis, n'ont cependant pas résisté aux tests sévères imposés par l'usage d'outils nouveaux. Dans ce sens, la mise au point de la technique de microélectrode chez l'animal en conditions chroniques (mesure de l'activité électrique d'un seul neurone) a permis de mettre en évidence, contrairement à ce qui était attendu, que les neurones sérotoninergiques sont actifs pendant l'éveil et que leur activité diminue pendant le sommeil a onde lente pour être totalement nulle pendant le sommeil paradoxal [6].

L'apport de cet outil soulignait sans ambiguilté que le niveau de compréhension des mécanismes neurochimiques du sommeil était insuffisant. Pour dépasser les contradictions soulevées par les techniques successivement utilisées, la mise au point d'outils plus performants que ceux disponibles s'avérait de nouveau nécessaire [7]. Dans ce contexte expérimental, la réalisation d'un outil permettant la mesure de la libération de neurotransmetteur, la voltamétrie, a été entreprise au début des années 80 [8], [9]. Le principe de cette methode consiste dans la mesure du courant d'oxydation produit par des substances oxydables et consécutif à l'application d'un potentiel variable. Ainsi, pour une valeur donnée du potentiel appliqué, s'oxyde une seule classe de composés, indissociable du point de vue électrochimique. Cet aspect constitue une limite vis-à-vis de la sélectivité des mesures. La méthode de dialyse intracrânienne n'est pas non plus parfaitement adapté pour la mesure des variations de neurotransmetteur à travers le cycle veillesommeil; dans ce sens, les délais temporels qui existent entre chaque mesure, al minimum de $5 \mathrm{~min}$, sont trop importants puisque les épisodes de SP ne durent que 2 minutes en moyenne. Sa résolution anatomique est de plus trop faible $(>1 \mathrm{~mm})$.

A ce stade expérimental et afin de progresser dans la compréhension des régulations qui assurent l'alternance du cycle éveil-sommeil, nous nous sommes orientés vers le développement de technologies alternatives. Afin de pouvoir adopter une solution technique adaptée, la mise au point d'un capteur chimique à fibre optique implantable dans les conditions hors anesthésie a été entreprise.

Dans cette optique les contraintes de départ du FOCS sont:

- le diamètre externe qui doit être inférieur à $300 \mu \mathrm{m}$ et à embout stérilizable;

- la possibilité de mesure chimique qui doit être rapide (inférieure à $30 \mathrm{~s}$ ) avec le meilleur rapport signal sur bruit, afin de réaliser une mesure sélective te suffisamment sensible. 
Pour la réalisation d'un FOCS qui permette de respecter ces contraintes, nous nous sommes orientés vers le développement d'une technique originale mettant en auvre des technologies de mesure de fluorescence induite par Laser UV, résolue en temps dans la gamme nanoseconde et autorisant une véritable signature moléculaire ainsi qu'une analyse quantitative des molécules mesurées. Le deuxième aspect important pour l'ingénierie du microcapteur est la réalisation d'un embout jetable biocompatible et stérilisable. Ce type de FOCS extrinsèque est actuellement fonctionnel et permet la mesure de fluorophores endogènes, comme le NADH mitochondrial.

\subsection{Mesure du NADH cérébral}

Le NADH est un composé chimique endogène impliqué dans la respiration cellulaire [1013]. Le couple $\mathrm{NADH} / \mathrm{NAD}^{+}$constitue le lien entre la dégradation des substrats et la formation d'ATP dans la chaîne des transferts électroniques $\left(P_{i}=\right.$ phosphore inorganique): (1) $\mathrm{SH}_{2}+\mathrm{NAD}^{+} \rightarrow \mathrm{S}+\mathrm{NADH}+\mathrm{H}^{+}$

$$
\text { (2) } \mathrm{NADH}+\mathrm{H}^{+}+3 \mathrm{ADP}+3 \mathrm{P}_{\mathrm{i}}+\frac{1}{2} \mathrm{O}_{2} \rightarrow \mathrm{NAD}^{+}+4 \mathrm{H}_{2} \mathrm{O}+3 \mathrm{ATP}
$$

La molécule de NAD comprend un cycle nicotinamide (noyau pyridine) lié à un cycle adénine par un pont diphosphorique (le NAD a été également appelé DPN DiphosphoPyridine Nucléotide, sa structure étant vue comme deux nucléotides -l'un formé d'adénine, ribose et phosphate, - l'autre de nicotinamide, ribose et phosphate):

La nicotinamide possède un ammonium quaternaire ce qui explique l'écriture sous la forme $\mathrm{NAD}^{+}$lorsqu'il est sous la forme oxydée. La forme réduite est dénommée NADH. Le NADH contient deux fluorophores: la partie 1,4 dihydronicotinamide et l'adénine. Cette partie 1,4 dihydronicotinamide lui confère des propriétés photophysiques différentes du NAD+ : le NADH en solution physiologique présente une bande d'absorption située sur $300-390 \mathrm{~nm}$ avec un maximum vers $340 \mathrm{~nm}$ et fluoresce vers 460 $\mathrm{nm}$. Le rendement quantique de fluorescence du NADH libre est de $2 \%$ [14]. Lorsqu'il est lié à un système enzymatique, son rendement augmente considérablement. Le NADH, suivant qu'il soit libre, lié à une protéine enzymatique ou en complexe ternaire (lié à une enzyme en présence d'un substrat), présente des variations du temps de déclin de fluorescence importantes pouvant passer de quelques centaines de picoseconde a $4 \mathrm{~ns}$ [15-17].

Les premières mesures fluorimétriques du NADH in vivo ont commencé en 1956 par l'étude de mitochondries isolées [10]. L'observation directe d'événements biochimiques intracellulaires et de leurs relations avec les fonctions physiologiques, restent encore d'un intéêtet indéniable [18-22]. Ces mêmes propriétés photophysiques ont été ensuite utilisées pour l'étude des variations du NADH in vivo du cerveau, limité souvent au cortex, [2332]. Il est connu que certains facteurs mécaniques et physiologiques peuvent modifier le signal de fluorescence mesuré in vivo. Dans la littérature [31], deux sources d'erreurs dans l'affectation des variations de l'intensité d'émission de fluorescence, liées aux seules variations du NADH, sont reportées, elles peuvent être liées:

-aux artefacts mécaniques dus aux mouvements respiratoires et aux pulsations cardiaques...

-aux changements de l'absorption et de la réflexion du tissu (artefacts oxymétriques survenant lors de changements du niveau d'oxygénation; artefacts hémodynamiques liés aux modifications du volume sanguin du tissu analysé).

Beaucoup d'auteurs suggerent que la modification principale du signal de fluorescence (autre que les variations dues aux NADH) est celle générée par les variations du volume sanguin de la zone analysee [33]. Les effets des modifications spectrales des pigments sanguins (hémoglobine), mitochondriaux (cytochromes, flavine) sur la fluorescence du $\mathrm{NAD}(\mathrm{P}) \mathrm{H}$ sont bien plus faibles [23], [31], [33]. L'approche dominante de ce probleme consiste à corriger le signal brut de fluorescence. Généralement le signal de fluorescence maximum est divisé par la mesure de la réflexion [27]. Cependant une autre école s'est formée pour tenir compte plus précisément des possibles artefacts oxymétriques: la fluorimétrie isobestique [29]. Selon cette technique les longueurs d'onde d'émission sont
choisies là où les hémoglobines oxygénées et désoxygénées présentent la même extinction moléculaire $(448 \mathrm{~nm}, 549 \mathrm{~nm}$ et $586 \mathrm{~nm})$. Le rapport de la fluorescence mesurée à $448 \mathrm{~nm}$ 
par la fluorescence enregistrée à $549 \mathrm{~nm}$ est alors considéré comme dénué d'artefacts oxymétriques et hémodynamiques. Les artefacts liés aux problèmes mécaniques sont les plus faciles à résoudre. L'utilisation de FO a permis un meilleur contrôle mécanique entre l'appareil de mesure et le tissu étudié. Les modalités des corrections hémodynamiques et oxymétriques dépendent étroitement des choix anatomiques, thématiques et instrumentaux des différents auteurs. Le choix anatomique (organes, tissus ...) détermine pour les mesures une sensibilité non négligeable aux variations du flux sanguin. Concernant l'oxygénation tissulaire, l'état d'oxydo-réduction mitochondrial nécessite des corrections des artefacts oxymétriques.

Actuellement au niveau de la litératture et pour le tissu cérébral, les choix anatomiques demeurent restreints à la surface du cerveau. Pour ces mesures realisées avec ou sans FO, la dure-mère et la pie-mère sont laissées intactes ou incisées sur des animaux anesthésiés et ventilés. Ces choix instrumentaux n'autorisent que des résolutions anatomiques millimétriques et nécessitent le maintien d'une distance entre le capteur et le tissu [25], [31]. Ainsi, il apparaît que la fluorimétrie tissulaire décrite au départ par Chance et al. [23] peut être mise en cuvre à travers une famille de systèmes distincts, tous fondés sur les mêmes propriétés photophysiques tissulaires. Chaque système reflète les compromis élaborés face aux choix techniques [29].

Nous avons montré précédemment [34] que la fluorescence mesurée à $460 \mathrm{~nm}$ ne provenait pas du liquide céphalo-rachidien mais principalement du compartiment intracellulaire. La mesure du NADH cérébral tissulaire intramitochondrial se révèle être intéressante non seulemnt au niveau du cortex mais aussi au niveau des noyaux. Nous n'avons pas relevé dans la littérature de travaux sur les tissus cérébraux profonds chez l'animal non anesthésié. Considérant les diverses approches ainsi que de leurs limites et persuadés que le potentiel de mesure fluorimétrique in vivo n'a été qu'effleuré, nous avons donc développé un microcapteur stérilisable offrant une résolution anatomique submillimétrique (diamètre de $200 \mu \mathrm{m}$ au stade de développement actuel) et autorisant des mesures sur des groupes cellulaires de taille réduite (nRD) dans des conditions physiologiques non traumatisantes (rat non anesthésié et libre de tous mouvements).

En utilisant les dernières évolutions de l'instrumentation des microcapteurs chimiques, un équipement de base afférent à ce capteur a été développé. Dès la fin des années quatrevingt, les capteurs chimiques à fibre optique ont commencé a utiliser la fluorescence résolue en temps soit en restant dans le domaine temporel soit par démodulation et déphasage [35-41]. Persuadés que la fluorescence résolue en temps est une technique adaptée aux FOCS en milieux complexes, nous avons réalisé un microcapteur basé sur la mesure résolue en temps et en longueur d'onde, de la fluorescence induite par excitation laser et émise par des substances présentes dans le tissu cérébral du rat non anesthésié. Ces mesures combinées avec la polygraphie classique ont permis de corréler les fluctuations de la fluorescence liée au NADH avec l'état de vigilance de l'animal.

\section{MATÉRIEL ET MÉTHODES}

\subsection{Procédure expérimentale:}

Sur des rats males OFA (IFFA.CREDO, $\mathrm{n}=4$ ) pesant $260-300 \mathrm{~g}$ anesthésiés au chloral hydrate $(400 \mathrm{mgr} / \mathrm{Kg})$, une cannule d'accès a été implantée dans le noyau du Raphé Dorsal (nRD) [42]. L'implantation des électrodes polygraphiques a été réalisée selon les méthodes déjà décrites [43]. Après dix jours de récupération, le FOCS étant placé dans la cannule, la mesure de fluorescence est mise en ouvre. Pendant un mois des sessions expérimentales de 6 heures par jour étaient réalisées sur le même animal. Les animaux étaient soumis à des cycles jour-nuit de $12 \mathrm{~h}-12 \mathrm{~h}$, a temperature constante et avec accès à l'eau et à la nourriture ad libitum.

Un control histologique de la position du FOCS a éte réalisé pour chaque animal. Le repérage de la position de la FO doit être réalisé par le passage d'un courant de $1 \mathrm{~mA} / 2 \mathrm{~s} a$ travers un fil conducteur de même longueur que la FO et mis en place dans la cannule guide.

2.2. Méthodes:

Le schéma expérimental est représenté figure 1. Ce montage a été décrit précédemment [44], [34]. La fibre optique utilisée est une PCS 200 de Quartz et Silice special UV. de diametre de cour $200 \mu \mathrm{m}$ et de diamètre extérieure $380 \mu \mathrm{m}$. 


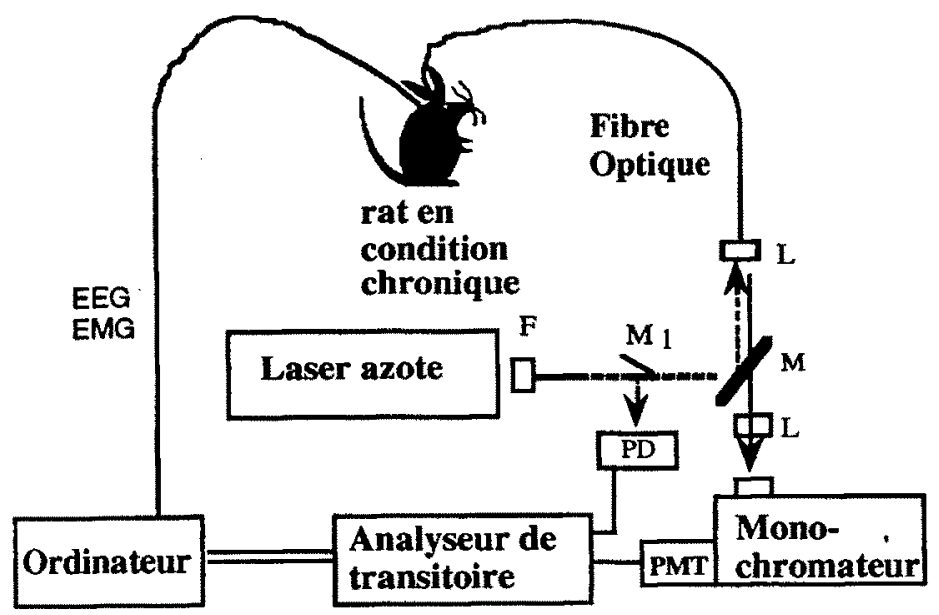

Figure 1. Représentation de l'instrumentation associée au FOCS: F, filtre; M, miroir dichroïque; Mi miroir; L,lentille; PMT, photomultiplicateur; PD, photodiode.

\section{RÉSULTATS ET DISCUSSION}

La Figure 2 présente l'ensemble des résultats pour les quatre rats enregistrés. Malgré de nombreuses questions sur la nature oxydative du métabolisme mis en jeu pendant le SP, aucune donnée sur les variations du NADH cérébral survenant au cours du cycle veillesommeil, n'ont été répertoriées dans la littérature. Jusqu'à présent, le NADH, n'est ni un neurotransmetteur ni un métabolite de neurotransmetteur. II est impliqué dans divers processus biochimiques. Ses variations spontanées a travers le cycle veille-sommeil sont faibles. En outre, des différences sur le sens des variations du signal enregistré à $460 \mathrm{~nm}$ par rapport à l'état de vigilance ont été enregistrees suivant les animaux (Figure 2). Ces différences pourraient provenir des sites de mesure, comme cela a été reporté pour la voltamétrie au niveau du $\mathrm{nRD}$ [45]:

- partie anterieure et ventrale du nRD, augmentation pendant le SL et le SP;

-zone antérieure et dorsale, augmentation seulement pendant le SP.

Avec le FOCS, une augmentation du signal a été effectivement obtenu chez 2 animaux dans la partie antérieure et ventrale du $\mathrm{NRD}$.

Comme pour le capteur voltamétrique, si le FOCS est situé à la limite de ce noyau (en particulier contre le troisième ventricule) ou à l'extérieur, des variations différentes ont été enregistrées pendant un même état de vigilance.

Au niveau du nRD les neurones sérotoninergiques sont largement présents. Chez le chat $77.5 \%$ des ces neurones sont localisés au niveau des noyaux du raphé dont $40.3 \%$ pour le $\mathrm{nRD}$ [46]. Des chiffres comparables ont été rapportés pour la souris [47]. II est aussi connu que les noyaux du raphé innervent pratiquement l'ensemble du système nerveux [48]. Les neurones sérotoninergiques deviennent progressivement inactifs pendant le sommeil lent pour devenir complètement silencieux pendant le sommeil paradoxal ; à travers ces deux stades de vigilance, la libération axonale de 5-HT est aussi diminuée [8]. L'activité électrique unitaire des neurones sérotoninergiques et la libération axonale de 5HT sont bien corrélées [49]. Ces deux parametres sont au minimum pendant le sommeil paradoxal puis augmentent pendant le sommeil lent et enfin atteignent un maximum pendant l'éveil. Au cours de ce processus d'inactivation des neurones serotoninergiques, la technique de voltamétrie a permis de mettre en évidence une libération de composés 5hydroxyindoliques au niveau des corps cellulaires du nRD; à l'inverse de la libération axonale, cette libération probablement dendritique est réduite pendant l'éveil puis augmente pendant le sommeil lent et atteint un maximum pendant le sommeil paradoxal [50]. A ces deux modalités de libération, axonale et dendritique, diachronique et synchronique du sommeil, correspondent en plus des rôles fonctionnels differents. La libération axonale est déjà connue pour être impliquée dans de nombreuses fonctions 
autres que celles du sommeil (régulation de la prise alimentaire, de l'activité sexuelle, de la douleur, de la température...). La libération dendritique intervient sur les fonctions veille-sommeil en étant en partie responsable de l'inhibition de l'activité électrique des neurones sérotoninergiques qui accompagne toujours le sommeil.

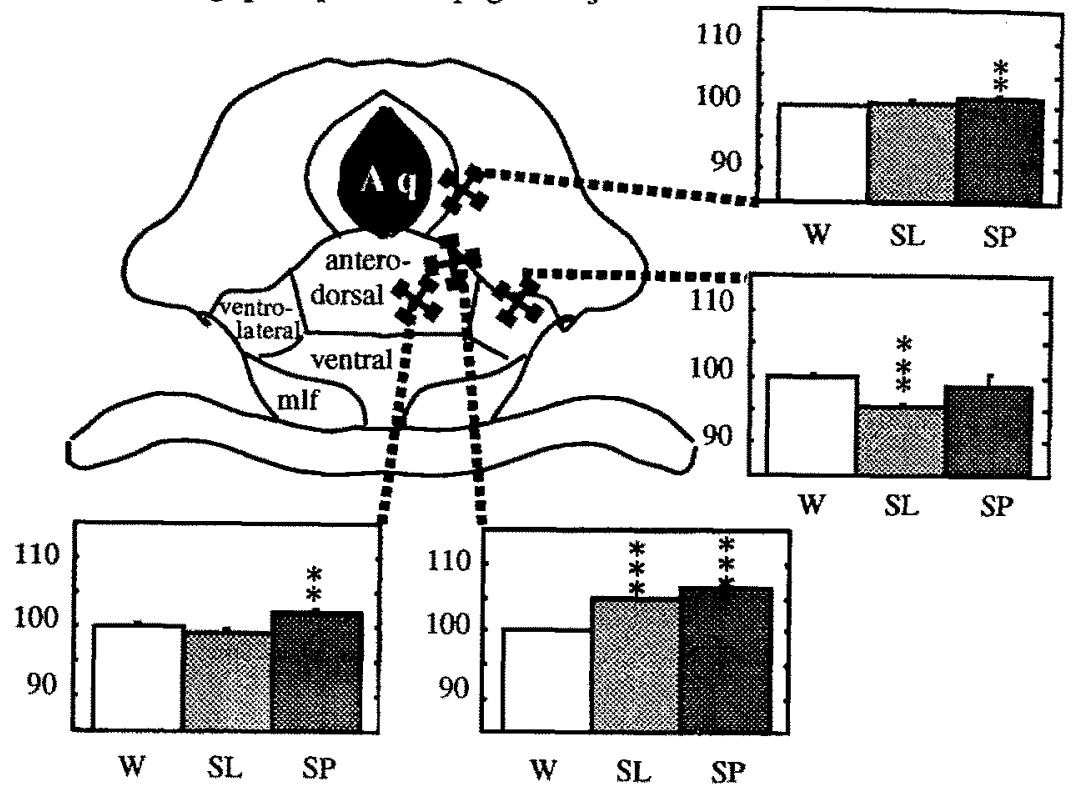

Figure 2. Fluorescence enregistrée a $460 \mathrm{~nm}$ in vivo au niveau du nRD sur quatre rats et moyenné par états (les barres représentent lerreur standard) avec l'eveil W (100\%), le sommeil a onde lente (SL) et le sommeil paradoxal (SP); chaque point correspond a 10 seconds d'integration du signal (nombre de points total pour W: 5000 ; pour SL: 4000 et pour SP: 1050). L'état de vigilance, déterminé par analyse visuelle des tracés polygraphiques est indiqué en bas de chaque bcolonne. Le niveau de significativité est obtenu par un test de $t: * P<0.05 ; * * P<0.01 ; * * * P<0.001$. La localisation du capteur dans le nRD est representee dans le plan frontal anterieur 1.00 (Atlas Paxinos et Watson). Aq: aqueduct; mfl: medial longitudinal fasciculus.

Au niveau du $\mathrm{Cx}$, le signal voltamétrique (corrélé avec le niveau de 5-HT) diminue pendant le SL et chute encore pendant le SP. Alors que pour le nRD, ce signal passe de $100 \%$ à l'éveil à $120 \%$ pendant le SL et $163 \%$ pendant le SP [51].

Au niveau du $\mathrm{nRD}$, d'autres neurones ou éléments non sérotoninergiques sont présents. Leur rôle dans les mécanismes de déclenchement et de maintien du sommeil sont suspectés, mais restent à préciser [52]. Compte tenu de ces données expérimentales, il apparaît que la liberation dendritique de 5-HT et l'intervention d'autres mécanismes non sérotoninergiques au niveau du $\mathrm{nRD}$ pourraient jouer un rôle important pour le déclenchement et le maintien du sommeil.

Pendant le SP, des oscillations d'amplitude importante de la demande en $\mathrm{O}_{2}$ ont été mesurées dans diverses parties cérébrales [53], [54]. Une augmentation du flux sanguin cérébral (CBF) pendant le SP a été rapportée [13]. Ces résultats ainsi que la nature du tracé EEG pendant le SP, suggèrent une augmentation du métabolisme oxydatif. Cependant, l'inactivation des neurones du nRD observée pendant le SP, qui résulte probablement de l'activation simultanée des dendrites, pose de nombreuses interrogations sur la situation énergétique du cerveau en tant qu'entité homogène.

Au niveau de la partie antérieure et ventrale du $\mathrm{nRD}$, une augmentation de la fluorescence $(460 \mathrm{~nm})$ a été aussi observé lors du SP par notre technique. Il semble évident de suggêrer que cette variation puisse reflêter l'activité dendritique des neurones sérotoninergiques du $\mathrm{nRD}$. Cette variation du NADH est cependant plus difficile à interpréter que celles d'un neurotransmetteur car ce composé intervient à plusieurs niveaux comme nous l'avons vu précedemment. L'élévation du niveau de NADH pendant 
le SP pourraient être consécutive à une augmentation de la réduction du $\mathrm{NAD}^{+}$(élévation du niveau des substrats $\mathrm{SH}_{2}$ ). Une diminution de l'oxydation du $\mathrm{NADH}$ (chute de l'accessibilité à $\mathrm{l}^{\prime} \mathrm{O}_{2}$ avec élévation du niveau d'ADP lié au degré de respiration mitochondriale) reste aussi probable.

\section{CONCLUSION}

Il faut souligner que pour la première fois, des variations physiologiques de composés endogènes ont été mesurées par des méthodes optiques, au niveau du nRD, chez des animaux en conditions chroniques et non anesthésiés. La mesure des variations de composés neurochimiques avec une bonne résolution anatomique $(200 \mu \mathrm{m})$ et une rapidité satisfaisante (10 secondes) a été réalisée. La sélectivité chimique est assurée par la longueur d'onde d'excitation $(337 \mathrm{~nm})$ et la longueur d'onde d'émission ( $460 \mathrm{~nm}+4 \mathrm{~nm})$. L'ensemble des données rapportées indiquent l'existence probable mais réservée d'un métabolisme oxydatif au niveau du $\mathrm{nRD}$, pouvant jouer un rôle dans les mécanismes de déclenchement et de maintien du sommeil.

Les résultats obtenus à l'aide de l'approche originale décrite, démontrent les fortes potentialités de celle-ci avec la mise en ouvre possible de nouveaux ensembles laserdétection plus performants.

Remerciements: les auteurs remercient la Lyonnaise de Banque (bourse post-doctorale de S. Mottin).

\section{Références}

[1] Mitchell D. G., Garden J. S., and Aldous K. M., Anal.Chem. 48 (1976) 2275-2277.

[2] Smith R. M., Jackson K. W., and Aldous K.M., Anal.Chem. 49 (1977) 2051-2053.

[3] Thompson R. B., Jones E. R., Anal.Chem. 65 (1993) 730-734.

[4] Benveniste H., J. Neurochem. 52 (1989) 1667-1679.

[5] Jouvet M., Science 163 (1969) 32-41.

[6] McGinty D. J., Harper R. M., Brain Research 101 (1976) 569-575.

[7] Ponchon J-L, Cespuglio R., Gonon F., Jouvet M., and Pujol J-F, Anal.Chem. 51 (1979) 1479-1482.

[8] Cespuglio R., Faradji H., Hahn Z., and Jouvet M., Measurement of neurotransmitter release (Wiley and Sons, 1983) 173-191.

[9] Cespuglio R., Sarda N., Gharib A., Chastrette N., Houdoin F., Rampin C., and Jouvet M., Exp. Brain.Res. 80 (1990) 121-128.

[10] Chance B., Williams G. R., Adv. Enzymol. 17 (1956) 65-134.

[11] Macllwain H., "Biochemistry and the central nervous system", (Churchill, J.\&A.,LTD., 1959) 110 \& 140-143 \& 256.

[12] Estabrook R. W., Anal. Biochem. 4 (1962) 231-245.

[13] Siesjö B. K., Brain Energy Metabolism (John Wiley \& sons, 1978) 187-270.

[14] Gafni A., Brand L., Biochemistry 15 (1976) 3165-3171.

[15] Brochon J. C., Wahl P., Jallon J. M., and Iwatsubo M., Biochemistry 15 (1976) 3259-3265.

[16] Lakowicz J. R., Szmacinski H., Nowaczyk K., and Johnson M. L., Proc. Nat. Acad.Sci.USA 89 (1992) 1271-1275.

[17] Couprie M. E., Mérola F., Tauc P., Garzella D., Delboulbé A., Hara T., and Billardon M., Rev.Sci.Instrum. 65 (1994) 1485-1495.

[18] Schramm W., Nittka M., Höhne W., and Kronfeld H-D, "Registrations of damages of endothelial cell cultures by NADH fluorescence", BiosEurope, Lille, 2329, (Proc. SPIE, 1994) sous presse.

[19] Krug A., Kessler M., Höper J., Batz M., Otto A., Zellner S., and Gärtner D., "Simultaneous monitoring of NAD(P)H, cytochromes, pO2 and $\mathrm{HbO} 2$ in liver tissue", BiosEurope, Lille, 2324, (Proc. SPIE, 1994) sous presse.

[20] Bocher T., Beuthan J., Minet O., Schmitt I., Fuchs B., and Müller G., "Fiberoptical sampling of NADH-concentration in Guinea-pig hearts during ischemia", BiosEurope, Lille, 2324, (Proc. SPIE, 1994) sous presse. [21] Schneckenburger H., Gschwend H., Paul R-J, Stepp H., Rick K., Betz V., and
Strauß W., "Time-gated spectroscopy of intrinsic fluorophores in cells and tissues",
BiosEurope. Lille BiosEurope, Lille, 2324, (Proc. SPIE, 1994) sous presse. 
[22] Betz V., Schneckenburger H., Alleröder H-P, Sybrecht G. W., and Meyer J-U, "Evaluation of changes in the NADH level between carcinogenic and normal tissue samples by use of fluorescence spectroscopy", Lille, 2324, (Proc. SPIE, 1994) sous presse.

[23] Chance B., Cohen P., Jobsis F., and Schoener B., Science 137 (1962) 499-508.

[24] Mayevsky A., Jamieson D., and Chance B., Brain Research 76 (1974) 481-491.

[25] Mayevsky A., Chance .B., Brain Research 98 (1975) 149-165.

[26] Lewis D. V., Schuette W. H., J.Neurophysiol. 38 (1975) 405-417.

[27] Harbig K., , Chance B., Kovach A. G. B., and Reivich M., J.Appl.Physiol. 41 (1976) 480-488.

[28] Ji S., Chance B., Stuart B. H., and Nathan R., Brain Research 119 (1977) 357. 373.

[29] Kramer R. S, Pearlstein R. D., Science 205 (1979) 693-696.

[30] Mayevsky A., Chance B., Science 217 (1982) 537-540.

[31] Mayevsky A., Brain Res. Rev. 7 (1984) 49-68.

[32] Hoshi Y., Tamura M., Brain Research 603 (1993) 215-221.

[33] Kobayashi S., Nishiki K., Kaede K., and Ogata E., J.Appl.Physiol. 31 (1971) 9396.

[34] Mottin S., Tran-Minh C., Laporte P., Cespuglio R., et Jouvet M., J. Phys. IV C4 (1994) 261-264.

[35] Vickers G. H., Miller R. M., and Hieftje G. M., Anal.Chim.Acta 192 (1987) 145153.

[36] Lippitsh M. E., Pusterhofer J., Leiner M. J. P., and Wolfbeis O. S., Anal.Chim.Acta 205 (1988) 1-6.

[37] DexpertGhys J., Halwani J., and Piriou B., Analusis 16 (1988) 81-86.

[38] Carroll M. K., Bright F. V., and Hieftje G. M., Anal.Chem. 61 (1989) 1768-1772.

[39] Varineau P. T., Duesing R., and Wangen L. E., Appl.Spectrosc. 45 (1991) 16521655 .

[40] Lakowicz J. R., Maliwal B., Anal.Chim.Acta 271 (1993) 155-164.

[41] Lakowicz J. R., Szmacinski H., and Karakelle M., Anal.Chim.Acta 272 (1993) 179-186.

[42] Paxinos G., Watsson C., "The rat brain in stereotaxic coordinates", (Academic Press, 1983) 85.

[43] Cespuglio R., Faradji H., and Jouvet M., In Monoamine innervation of cerebral cortex (Alain R. Liss Inc., 1984)

[44] Mottin S., Tran-Minh C., Laporte P., Cespuglio R., and Jouvet M., Appl.Spectrosc. 47 (1993) 590-597.

[45] Houdouin, F., "Les modalités de libération de la sérotonine à travers le cycle veillesommeil du rat. Rôles dans la préparation, le déclenchement et le maintien du sommeil.", thèse de l'Université Claude Bernard-Lyon1 (1991).

[46] Wiklund L., Léger L., and Persson M., J.Comp.Neurol. 203 (1981) 613-647.

[47] Takeushi Y., "Distribution of serotonin neurons in the mammalian brain", Neuronal Serotonin (Wiley J. and Sons, 1988) 25-56.

[48] Steinbush H. W. M., "Serotoninergic neurons in the central nervous system of the rat", (Nijmegen, 1982) 272-272.

[49] Fornal C. A., Jacobs B. L., Neuronal Serotonin (Wiley J. and Sons, 1988) 305345.

[50] Cespuglio R., Chastrette N., et Jouvet M., C.R.Acad.Sc. Paris 307 (1988) 817 823.

[51] Cespuglio R., Houdoin F., Oulerich M., El Mansari M., and Jouvet M., J.Sleep Res. 1 (1992) 150-156.

[52] Cespuglio R., Gomez M. E., Walker E., and Jouvet M., EEG Clin. Neurophysiol. 47 (1979) 289-308.

[53] GarciaAustt E., Velluti R., and Villar J. I., Physiol. Behav. 3 (1968) 477-485.

[54] Velluti R., Physiol. Behav. 34 (1985) 355-358. 